\title{
Tunnelling of Massive/Massless Bosons from the Apparent Horizon of FRW Universe
}

\author{
Kimet Jusufi, ${ }^{1,2}$ Ali Ovgun, ${ }^{3,4}$ and Gordana Apostolovska ${ }^{1}$ \\ ${ }^{1}$ Institute of Physics, Faculty of Natural Sciences and Mathematics, Ss. Cyril and Methodius University, Arhimedova 3, \\ 1000 Skopje, Macedonia \\ ${ }^{2}$ Physics Department, State University of Tetovo, Ilinden Street nn, 1200 Tetovo, Macedonia \\ ${ }^{3}$ Instituto de Física, Pontificia Universidad Católica de Valparaíso, Casilla 4950, Valparaíso, Chile \\ ${ }^{4}$ Physics Department, Eastern Mediterranean University, Famagusta, Northern Cyprus, Mersin 10, Turkey
}

Correspondence should be addressed to Kimet Jusufi; kimet.jusufi@unite.edu.mk

Received 5 April 2017; Accepted 22 May 2017; Published 3 July 2017

Academic Editor: Torsten Asselmeyer-Maluga

Copyright (C) 2017 Kimet Jusufi et al. This is an open access article distributed under the Creative Commons Attribution License, which permits unrestricted use, distribution, and reproduction in any medium, provided the original work is properly cited. The publication of this article was funded by SCOAP S $^{3}$

\begin{abstract}
We investigate the Hawking radiation of vector particles from the apparent horizon of a Friedmann-Robertson-Walker (FRW) universe in the framework of quantum tunnelling method. Furthermore we use Proca equation, a relativistic wave equation for a massive/massless spin-1 particle (massless $\gamma$ photons, weak massive $W^{ \pm}$and $Z^{0}$ bosons, strong massless gluons, and $\rho$ and $\omega$ mesons) together with a Painlevé space-time metric for the FRW universe. We solve the Proca equation via Hamilton-Jacobi (HJ) equation and the WKB approximation method. We recover the same result for the Hawking temperature associated with vector particles as in the case of scalar and Dirac particles tunnelled from outside to the inside of the apparent horizon in a FRW universe.
\end{abstract}

\section{Introduction}

In early 1970s, Hawking came with an idea that black holes emit thermal radiation because of the quantum effects near the event horizon [1-3]. It means that black holes are not completely black. This may meet an interesting experimental base for searching the vertex of general relativity and quantum mechanics. Unfortunately, the temperatures of Hawking radiation from black holes are far too small to be observed within the near future.

Since Hawking, many derivations of this radiation have been made. Most famous derivation is made by Parikh and Wilczek in which they use a tunnelling process with a semiclassical WKB approximation [4-12]. A number of important contributions have been done using different types of spherically symmetric and stationary black holes, for different types fields and corresponding particles such as scalar as well as for Dirac particles [13-26].

Besides the progress in the field, there are a number of conceptual problems related to Hawking radiation. For example, the information loss paradox remains still unsolved.
Furthermore there is no agreement among physicists whether this radiation is produced near the event horizon or in some larger region surrounding the black hole (see, e.g., [27]). If we assume that Hawking quanta are located very close to the event horizon, there is a problem when considering the infinite blueshift. In fact, it is interesting to note that, according to the tunnelling method, this process took place near the event horizon. However, recent studies show that quantum gravity effects and the nature of particles may affect the Hawking temperature. That is to say, when the GUP effects are introduced, the Hawking quanta near the event horizon can never be infinite-blue shifted due to the existence of minimal length [28].

Recently the quantum tunnelling of scalar and Dirac particles from the apparent horizon in a FRW universe has been investigated in [29-32]. On the other hand, since the famous thermodynamical derivation of Einstein's field equation by Jacobson [33], thermodynamical aspects of the apparent horizon in for FRW universe have been widely studied in the context of different gravity theories [34]. In this paper, we shall extend the work of Cai et al. [31] to explore 
the Hawking radiation of massive/massless spin-1 particles in the framework of quantum tunnelling method to compute Hawking temperature from the apparent horizon of a FRW universe.

This paper consists of three main parts. In Section 2, we give a brief review and introduce a Painlevé-type space-time metric for the FRW universe and then investigate the Hawking radiation of massive bosons via tunnelling. In Section 3, we apply the same method, namely, the $\mathrm{HJ}$ equation to the massless Hawking quanta (photon particles), and derive the Hawking temperature from the apparent horizon of a FRW universe. Finally, in the last part, we conclude our paper.

\section{Hawking Radiation of Massive Bosons via Tunnelling}

The FRW universe can be described by the following metric:

$$
d s^{2}=-d t^{2}+a^{2}(t)\left(\frac{d r^{2}}{1-k r^{2}}+r^{2} d \theta^{2}+r^{2} \sin ^{2} \theta d \varphi^{2}\right),
$$

in which $a(t)$ is the scale factor and $k$ encodes the geometry of FRW universe. In particular, $k=-1,0,1$ corresponds to a closed, flat, and open universe, respectively. Introducing a new coordinate transformation $\widetilde{r}=r a$, the above metric reduces to

$$
d s^{2}=h_{a b} d x^{a} d x^{b}+\tilde{r}^{2}\left(d \theta^{2}+\sin ^{2} \theta d \varphi^{2}\right) .
$$

From this equation we can define the apparent horizon as follows:

$$
h^{a b} \partial_{a} \tilde{r} \partial_{b} \tilde{r}=0
$$

Then one can show the following relation for the apparent horizon:

$$
\tilde{r}_{A}=\frac{1}{\sqrt{H^{2}+k / a^{2}}}
$$

where $H=\dot{a} / a$. Using $\tilde{r}=r a$, one can find Painlevé-type coordinates for FRW universe metric as follows:

$$
\begin{aligned}
d s^{2}= & -\frac{1-\widetilde{r}^{2} / \widetilde{r}_{A}^{2}}{1-k \widetilde{r}^{2} / a^{2}} d t^{2}-\frac{2 H \widetilde{r}}{1-k \widetilde{r}^{2} / a^{2}} d t d \widetilde{r} \\
& +\frac{1}{1-k \widetilde{r}^{2} / a^{2}} d \widetilde{r}^{2}+\widetilde{r}^{2}\left(d \theta^{2}+\sin ^{2} \theta d \varphi^{2}\right) .
\end{aligned}
$$

Later on, we shall use this metric to study the tunnelling of massive/massless bosons. Particles are nothing else but excitations of some fields; in particular vector particles are excitations of vector fields $\Psi^{\mu}$ which are described by the relativistic wave equation known as Proca equation

$$
\frac{1}{\sqrt{-g}} \partial_{\mu}\left(\sqrt{-g} \Psi^{\nu \mu}\right)+\frac{m^{2}}{\hbar^{2}} \Psi^{\nu}=0,
$$

and in order to solve the Proca equation we shall employ the following ansatz for our vector field $\Psi^{\nu}$ as follows:

$$
\Psi_{\mu}=C_{\mu} \exp \left(\frac{i}{\hbar} S(t, \tilde{r}, \theta, \varphi)\right) .
$$

On the other hand an observer will detect Hawking quanta. Before we shall go on to separate the variables in the action for the Hawking quanta one must consider the Kodama vector. The physical significance of the Kodama vector is related to the energy of those Hawking quanta measured by the Kodama observer

$$
\omega=-K^{a} \partial_{a} S=-\sqrt{1-\frac{k \widetilde{r}^{2}}{a^{2}}} \partial_{t} S,
$$

in which the Kodama vector is defined via

$$
K^{a}=\sqrt{1-\frac{k \tilde{r}^{2}}{a^{2}}}\left(\frac{\partial}{\partial t}\right)^{a} .
$$

In terms of the above relations one may choose the following form to the action for the Hawking quanta:

$$
S(t, \tilde{r}, \theta, \varphi)=-\int \frac{\omega}{\sqrt{1-k \widetilde{r}^{2} / a^{2}}} d t+R(\widetilde{r})+P(\theta, \varphi) .
$$

Substituting our action (10) into (6) and (7) in a Painlevétype space-time metric (5) for the FRW universe, we find the following set of four differential equations:

$$
\begin{aligned}
0= & -\left[\frac{g(\widetilde{r}) \sin ^{2} \theta\left(\partial_{\theta} P\right)^{2}+g(\widetilde{r})\left(\partial_{\varphi} P\right)^{2}+\sin ^{2} \theta \widetilde{r}^{2}\left(g(\widetilde{r}) m^{2}-t E(\widetilde{r}) E(\widetilde{r})^{\prime}+E(\widetilde{r}) R(\widetilde{r})^{\prime}\right)}{\Delta(\widetilde{r}) \sin ^{2} \theta \widetilde{r}^{2}}\right] C_{1} \\
& -\left[\frac{g(\widetilde{r}) E(\widetilde{r})^{\prime} t-g(\widetilde{r}) R(\widetilde{r})^{\prime}+h(\widetilde{r}) E(\widetilde{r})}{\Delta(\widetilde{r}) \widetilde{r}^{2}}\right]\left(\partial_{\theta} P\right) C_{2}-\left[\frac{g(\widetilde{r}) E(\widetilde{r})^{\prime} t-g(\widetilde{r}) R(\widetilde{r})^{\prime}+h(\widetilde{r}) E(\widetilde{r})}{\Delta(\widetilde{r}) \sin ^{2} \theta \widetilde{r}^{2}}\right]\left(\partial_{\varphi} P\right) C_{3} \\
& -\left[\frac{h(\widetilde{r}) \sin ^{2} \theta\left(\partial_{\theta} P\right)^{2}+h(\widetilde{r})\left(\partial_{\varphi} P\right)^{2}+\sin ^{2} \theta \widetilde{r}^{2}\left(t^{2} E(\widetilde{r})^{\prime 2}+h(\widetilde{r}) m^{2}-2 t R(\widetilde{r})^{\prime} E(\widetilde{r})^{\prime}+R(\widetilde{r})^{\prime 2}\right)}{\Delta(\widetilde{r}) \sin ^{2} \theta \widetilde{r}^{2}}\right] C_{4},
\end{aligned}
$$




$$
\begin{aligned}
& 0=\left[\frac{f(\widetilde{r}) \sin ^{2} \theta\left(\partial_{\theta} P\right)^{2}+f(\widetilde{r})\left(\partial_{\varphi} P\right)^{2}+\sin ^{2} \theta \widetilde{r}^{2}\left(f(\widetilde{r}) m^{2}-E(\widetilde{r})^{2}\right)}{\Delta(\widetilde{r}) \sin ^{2} \theta \widetilde{r}^{2}}\right] C_{1} \\
& +\left[\frac{f(\widetilde{r}) E(\widetilde{r})^{\prime} t-f(\widetilde{r}) R(\widetilde{r})^{\prime}-g(\widetilde{r}) E(\widetilde{r})}{\Delta(\widetilde{r}) \widetilde{r}^{2}}\right]\left(\partial_{\theta} P\right) C_{2}+\left[\frac{f(\widetilde{r}) E(\widetilde{r})^{\prime} t-f(\widetilde{r}) R(\widetilde{r})^{\prime}-g(\widetilde{r}) E(\widetilde{r})}{\Delta(\widetilde{r}) \sin ^{2} \theta \widetilde{r}^{2}}\right]\left(\partial_{\varphi} P\right) C_{3} \\
& -\left[\frac{g(\widetilde{r}) \sin ^{2} \theta\left(\partial_{\theta} P\right)^{2}+g(\widetilde{r})\left(\partial_{\varphi} P\right)^{2}+\sin ^{2} \theta \widetilde{r}^{2}\left(g(\widetilde{r}) m^{2}-t E(\widetilde{r}) E(\widetilde{r})^{\prime}+E(\widetilde{r}) R(\widetilde{r})^{\prime}\right)}{\Delta(\widetilde{r}) \sin ^{2} \theta \widetilde{r}^{2}}\right] C_{4}, \\
& 0=\left[\frac{f(\widetilde{r}) E(\widetilde{r})^{\prime} t-f(\widetilde{r}) R(\widetilde{r})^{\prime}-g(\widetilde{r}) E(\widetilde{r})}{\Delta(\widetilde{r}) \widetilde{r}^{2}}\right]\left(\partial_{\theta} P\right) C_{1} \\
& +\left[\frac{\Delta\left(\partial_{\theta} P\right)^{2}+\sin ^{2} \theta \widetilde{r}\left(t^{2} f\left(E(\widetilde{r})^{\prime}\right)^{2}-2 t E(\widetilde{r})^{\prime}\left(g E+f R(\widetilde{r})^{\prime}\right)+m^{2} \Delta+2 g E R(\widetilde{r})^{\prime}-E^{2} h+f\left(R(\widetilde{r})^{\prime}\right)^{2}\right)}{\Delta(\widetilde{r}) \sin ^{2} \theta \widetilde{r}^{4}}\right] C_{2} \\
& -\frac{\left(\partial_{\theta} P\right)\left(\partial_{\varphi} P\right)}{r^{4} \sin ^{2} \theta} C_{3}+\left[\frac{g(\widetilde{r}) E(\widetilde{r})^{\prime} t-g(\widetilde{r}) R(\widetilde{r})^{\prime}+h(\widetilde{r}) E(\widetilde{r})}{\Delta(\widetilde{r}) \tilde{r}^{2}}\right]\left(\partial_{\theta} P\right) C_{4}, \\
& 0=\left[\frac{f(\widetilde{r}) E(\widetilde{r})^{\prime} t-f(\widetilde{r}) R(\widetilde{r})^{\prime}-g(\widetilde{r}) E(\widetilde{r})}{\Delta(\widetilde{r}) \sin ^{2} \theta \widetilde{r}^{2}}\right]\left(\partial_{\varphi} P\right) C_{1}-\frac{\left(\partial_{\theta} P\right)\left(\partial_{\varphi} P\right)}{r^{4} \sin ^{2} \theta} C_{2} \\
& +\left[\frac{\Delta\left(\partial_{\theta} P\right)^{2}+\widetilde{r}\left(t^{2} f\left(E(\widetilde{r})^{\prime}\right)^{2}-2 t E(\widetilde{r})^{\prime}\left(g E+f R(\widetilde{r})^{\prime}\right)+m^{2} \Delta+2 g E R(\widetilde{r})^{\prime}-E^{2} h+f\left(R(\widetilde{r})^{\prime}\right)^{2}\right)}{\Delta(\widetilde{r}) \sin ^{2} \theta \widetilde{r}^{4}}\right] C_{3} \\
& -\left[\frac{g(\widetilde{r}) E(\widetilde{r})^{\prime} t-g(\widetilde{r}) R(\widetilde{r})^{\prime}+h(\widetilde{r}) E(\widetilde{r})}{\Delta(\widetilde{r}) \sin ^{2} \theta \widetilde{r}^{2}}\right]\left(\partial_{\varphi} P\right) C_{4},
\end{aligned}
$$

where we have introduced

$$
\begin{aligned}
& f(\widetilde{r})=\frac{1-\widetilde{r}^{2} / \widetilde{r}_{A}^{2}}{1-k \widetilde{r}^{2} / a^{2}}, \\
& g(\widetilde{r})=\frac{H \widetilde{r}}{1-k \widetilde{r}^{2} / a^{2}}, \\
& h(\widetilde{r})=\frac{1}{1-k \widetilde{r}^{2} / a^{2}},
\end{aligned}
$$

$$
\begin{aligned}
& E(\widetilde{r})=\frac{\omega}{\sqrt{1-k \widetilde{r}^{2} / a^{2}}}, \\
& \Delta(\widetilde{r})=f(\widetilde{r}) h(\widetilde{r})+g(\widetilde{r})^{2} .
\end{aligned}
$$

Then by using a $4 \times 4$ matrix $\Xi$ and a vector $\left(C_{1}, C_{2}, C_{3}, C_{4}\right)$ the following matrix equation is satisfied:

$$
\Xi\left(C_{1}, C_{2}, C_{3}, C_{4}\right)^{T}=0 .
$$

Moreover this matrix is characterised by the following nonzero matrix elements:

$$
\begin{aligned}
& \Xi_{11}=\Xi_{24}=-\frac{g(\widetilde{r}) \sin ^{2} \theta\left(\partial_{\theta} P\right)^{2}+g(\widetilde{r})\left(\partial_{\varphi} P\right)^{2}+\sin ^{2} \theta \widetilde{r}^{2}\left(g(\widetilde{r}) m^{2}-t E(\widetilde{r}) E(\widetilde{r})^{\prime}+E(\widetilde{r}) R(\widetilde{r})^{\prime}\right)}{\Delta(\widetilde{r}) \sin ^{2} \theta \widetilde{r}^{2}}, \\
& \Xi_{12}=-\Xi_{34}=-\left[\frac{g(\widetilde{r}) E(\widetilde{r})^{\prime} t-g(\widetilde{r}) R(\widetilde{r})^{\prime}+h(\widetilde{r}) E(\widetilde{r})}{\Delta(\widetilde{r}) \widetilde{r}^{2}}\right]\left(\partial_{\theta} P\right),
\end{aligned}
$$




$$
\begin{aligned}
& \Xi_{13}=\Xi_{44}=-\left[\frac{g(\widetilde{r}) E(\widetilde{r})^{\prime} t-g(\widetilde{r}) R(\widetilde{r})^{\prime}+h(\widetilde{r}) E(\widetilde{r})}{\Delta(\widetilde{r}) \sin ^{2} \theta \widetilde{r}^{2}}\right]\left(\partial_{\varphi} P\right), \\
& \Xi_{14}=-\frac{h(\widetilde{r}) \sin ^{2} \theta\left(\partial_{\theta} P\right)^{2}+h(\widetilde{r})\left(\partial_{\varphi} P\right)^{2}+\sin ^{2} \theta \widetilde{r}^{2}\left(t^{2} E(\widetilde{r})^{\prime 2}+h(\widetilde{r}) m^{2}-2 t R(\widetilde{r})^{\prime} E(\widetilde{r})^{\prime}+R(\widetilde{r})^{\prime 2}\right)}{\Delta(\widetilde{r}) \sin ^{2} \theta \widetilde{r}^{2}}, \\
& \Xi_{21}=\frac{f(\widetilde{r}) \sin ^{2} \theta\left(\partial_{\theta} P\right)^{2}+f(\widetilde{r})\left(\partial_{\varphi} P\right)^{2}+\sin ^{2} \theta \widetilde{r}^{2}\left(f(\widetilde{r}) m^{2}-E(\widetilde{r})^{2}\right)}{\Delta(\widetilde{r}) \sin ^{2} \theta \widetilde{r}^{2}}, \\
& \Xi_{22}=\Xi_{31}=\left[\frac{f(\widetilde{r}) E(\widetilde{r})^{\prime} t-f(\widetilde{r}) R(\widetilde{r})^{\prime}-g(\widetilde{r}) E(\widetilde{r})}{\Delta(\widetilde{r}) \widetilde{r}^{2}}\right]\left(\partial_{\theta} P\right), \\
& \Xi_{23}=\Xi_{41}=\left[\frac{f(\widetilde{r}) E(\widetilde{r})^{\prime} t-f(\widetilde{r}) R(\widetilde{r})^{\prime}-g(\widetilde{r}) E(\widetilde{r})}{\Delta(\widetilde{r}) \sin ^{2} \theta \widetilde{r}^{2}}\right]\left(\partial_{\varphi} P\right), \\
& \Xi_{43}=\frac{\Delta\left(\partial_{\theta} P\right)^{2}+\widetilde{r}\left(t^{2} f\left(E(\widetilde{r})^{\prime}\right)^{2}-2 t E(\widetilde{r})^{\prime}\left(g E+f R(\widetilde{r})^{\prime}\right)+m^{2} \Delta+2 g E R(\widetilde{r})^{\prime}-E^{2} h+f\left(R(\widetilde{r})^{\prime}\right)^{2}\right)}{\Delta\left(\partial_{\theta} P\right)^{2}+\sin ^{2} \theta \widetilde{r}\left(t^{2} f\left(E(\widetilde{r})^{\prime}\right)^{2}-2 t E(\widetilde{r})^{\prime}\left(g E+f R(\widetilde{r})^{\prime} \theta \widetilde{r}^{4}+m^{2} \Delta+2 g E R(\widetilde{r})^{\prime}-E^{2} h+f\left(R(\widetilde{r})^{\prime}\right)^{2}\right)\right.} \\
& \Xi_{32}=\frac{\left(\partial_{\theta} P\right)\left(\partial_{\varphi} P\right)}{r^{4} \sin ^{2} \theta}, \\
& \Xi_{42}=-\sin ^{2} \theta \widetilde{r}^{4}
\end{aligned}
$$

In order to see the physical significance of these equations let us first solve $\operatorname{det} \Xi=0$, to find the following equation:

$$
\begin{aligned}
& \frac{1}{\sin ^{8} \theta \Delta(\widetilde{r}) \widetilde{r}^{12}}\left[\left(\left(m^{2} \sin ^{2} \theta \widetilde{r}^{2}+\left(\partial_{\varphi} P\right)^{2}\right) \Delta(\widetilde{r})\left(\partial_{\theta} P\right)^{2}\right)\right. \\
& +42 m \Delta(\widetilde{r}) \widetilde{r}^{4} \sin ^{2} \theta\left(\partial_{\theta} P\right)\left(\partial_{\varphi} P\right)+m^{2}(\Delta(\widetilde{r}) \\
& \left.\cdot\left(\partial_{\varphi} P\right)^{2}+\sin ^{2} \theta \widetilde{r}^{2} \zeta(\widetilde{r}) r^{2}\right)\left(\Delta(\widetilde{r}) \sin ^{2} \theta\left(\partial_{\theta} P\right)^{2}\right. \\
& \left.\left.+\Delta(\widetilde{r})\left(\partial_{\varphi} P\right)^{2}+\sin ^{2} \theta \widetilde{r}^{2} \zeta(\widetilde{r})\right)^{2}\right]=0
\end{aligned}
$$

Solving (15) for the radial part, we find the following solution:

$$
R(\widetilde{r})_{ \pm}=\frac{\omega}{\sqrt{1-k \widetilde{r}^{2} / a^{2}}} \int d t-\int \frac{g(\widetilde{r}) E(\widetilde{r}) \pm \sqrt{g(\widetilde{r})^{2} E(\widetilde{r})^{2}-f(\widetilde{r})\left[\Delta(\widetilde{r})\left(m^{2}+\left(\partial_{\theta} P\right)^{2}+\left(\partial_{\varphi} P\right)^{2}\right)-h(\widetilde{r}) E(\widetilde{r})^{2}\right]}}{f(\widetilde{r})} d \widetilde{r}
$$

and we note that +/- sign in the last equation corresponds to the incoming (outgoing) wave solutions. At the apparent horizon $\widetilde{r}=\widetilde{r}_{A}$, it is clear that the function $f(\widetilde{r})$ vanishes; that is, $f\left(\widetilde{r}_{A}\right)=0$. Solving this integral we see that there is a zero contribution to the imaginary part coming from the first term; on the other hand solving the second integral we find

$$
\begin{aligned}
& \operatorname{Im} R_{+}=\pi \omega \widetilde{r}_{A}, \\
& \operatorname{Im} R_{-}=0 .
\end{aligned}
$$


Hence, we have shown that there is no contribution of an outgoing particle to the imaginary part of the action. This shows that the tunnelling of Hawking quanta is from the outside to the inside of the apparent horizon which is different from the black hole case. Finally, the tunnelling probability can be calculated as [29]

$$
\begin{aligned}
\Gamma & =\frac{P_{\text {in }}}{P_{\text {out }}}=\frac{\exp \left[-2\left(\operatorname{Im} R_{+}+\operatorname{Im} P\right)\right]}{\exp \left[-2\left(\operatorname{Im} R_{-}+\operatorname{Im} P\right)\right]} \\
& =\exp \left(-2 \pi \omega \widetilde{r}_{A}\right) .
\end{aligned}
$$

Using the last result and comparing to the Boltzmann equation $\Gamma=\exp \left(-\omega / T_{H}\right)$, we recover the Hawking temperature from the apparent horizon

$$
T_{H}=\frac{1}{2 \pi \widetilde{r}_{A}}
$$

Hence, we have successfully derived the same relation for the Hawking temperature as in the case of scalar and Dirac particles tunnelled from the apparent horizon of FRW universe [29-32]. We point out that this thermal flux of radiation with a temperature $T_{H}$ can be detected by Kodama observer which is inside the apparent horizon.

\section{Hawking Radiation of Photons from FRW Universe}

In this section, we shall focus on tunnelling of photon particles using the HJ method $[35,36]$. The action without the gauge fixing term is given as follows:

$$
S=-\frac{1}{4} \int F_{\mu \nu} F^{\mu \nu} \sqrt{-g} d^{4} x
$$

and then the equation of motion for this Maxwell field is derived as

$$
\nabla^{\mu} F_{\mu \nu}=0
$$

where the field strength $F_{\mu \nu}$ is given in terms of the gauge field $A_{\mu}$ as follows:

$$
F_{\mu \nu}=\nabla_{\mu} A_{\nu}-\nabla_{\nu} A_{\mu}
$$

using the Lorentz gauge condition. Now, we use the same procedure, and first we define the following ansatz for photon field (Maxwell equation):

$$
A_{\mu}=C_{\mu} \exp \left(\frac{i}{\hbar} S(t, \tilde{r}, \theta, \varphi)\right)
$$

Again the energy of those quanta (photons) measured by the Kodama observer reads

$$
\omega=-K^{a} \partial_{a} S=-\sqrt{1-\frac{k \widetilde{r}^{2}}{a^{2}}} \partial_{t} S,
$$

where the Kodama vector is defined via

$$
K^{a}=\sqrt{1-\frac{k \tilde{r}^{2}}{a^{2}}}\left(\frac{\partial}{\partial t}\right)^{a} .
$$

We choose the following action for the tunnelling quanta:

$$
S(t, \tilde{r}, \theta, \varphi)=-\int \frac{\omega}{\sqrt{1-k \tilde{r}^{2} / a^{2}}} d t+R(\widetilde{r})+P(\theta, \varphi) \text {. }
$$

After we solve Maxwell's equations on the background of FRW universe in Painlevé space-time we solve for $\operatorname{det} \Xi=$ 0 , and then we find the following radial part for the wave solution:

$$
R(\widetilde{r})_{ \pm}=\frac{\omega}{\sqrt{1-k \widetilde{r}^{2} / a^{2}}} \int d t-\int \frac{g(\widetilde{r}) E(\widetilde{r}) \pm \sqrt{g(\widetilde{r})^{2} E(\widetilde{r})^{2}-f(\widetilde{r})\left[\Delta(\widetilde{r})\left(\left(\partial_{\theta} P\right)^{2}+\left(\partial_{\varphi} P\right)^{2}\right)-h(\widetilde{r}) E(\widetilde{r})^{2}\right]}}{f(\widetilde{r})} d \widetilde{r}
$$

By repeating the same argument as in the last section we end up with exactly the same expressions for the imaginary part

$$
\begin{aligned}
& \operatorname{Im} R_{+}=\pi \omega \widetilde{r}_{A}, \\
& \operatorname{Im} R_{-}=0 .
\end{aligned}
$$

Using the Boltzmann formula $\Gamma=\exp \left(-\omega / T_{H}\right)$, we find the same result for the Hawking temperature

$$
T_{H}=\frac{1}{2 \pi \widetilde{r}_{A}}
$$

Hence, we have concluded that the temperature of the Hawking radiation associated with the apparent horizon does not depend on the mass or type of tunnelling particles. It took Jacobson to show that Einstein's field equations can be interpreted as a thermodynamic law $T d S=d E+P d V$ [33]. Later on, this idea was put forward by many authors; in $[37,38]$ this idea was generalised to the Friedmann equation for FRW universe. Among other things, it was shown that Friedmann equation can also be viewed in terms of the first law of thermodynamics $d E=T d S+W d V$, at the apparent horizon. Note that $W=(\rho-P) / 2$ is the work density, $E$ is the energy, $V$ gives the volume inside the apparent horizon, $\rho$ is energy density, and $P$ is pressure of matter in the universe. Recently in [34] a modified Friedmann equation with a power-law corrected entropy was investigated. We plan in the near future to investigate the role of entanglement of tunnelling particles from the apparent horizon. 


\section{Conclusion}

In this article we have investigated the Hawking radiation of massive/massless vector particles in the framework of quantum tunnelling method from the apparent horizon of FRW universe. We have solved the relativistic Proca equation for massive and massless Hawking quanta working in a Painlevétype space-time metric for FRW universe. In the last part we have found the radial solution of the wave function using the WKB approximation method and the Kodama vector. We have shown that quantum tunnelling of massive/massless vector particles from the apparent horizon of FRW universe gives rise to the same expression for the Hawking temperature as in the case of scalar or Dirac particles.

\section{Conflicts of Interest}

The authors declare that there are no conflicts of interest regarding the publication of this paper.

\section{Acknowledgments}

This work was supported by the Chilean FONDECYT Grant no. 3170035 (AÖ).

\section{References}

[1] S. W. Hawking, "Particle creation by black holes," Communications in Mathematical Physics, vol. 43, no. 3, pp. 199-220, 1975.

[2] G. W. Gibbons and S. W. Hawking, "Action integrals and partition functions in quantum gravity," Physical Review D, vol. 15, no. 10 , pp. 2752-2756, 1977.

[3] G. W. Gibbons and S. W. Hawking, "Cosmological event horizons, thermodynamics, and particle creation," Physical Review. D. Particles and Fields. Third Series, vol. 15, no. 10, pp. 2738-2751, 1977.

[4] M. K. Parikh and F. Wilczek, "Hawking radiation as tunneling," Physical Review Letters, vol. 85, no. 24, pp. 5042-5045, 2000.

[5] M. K. Parikh, "New coordinates for de Sitter space and de Sitter radiation," Physics Letters B, vol. 546, no. 3-4, pp. 189-195, 2002.

[6] M. Parikh, "A secret tunnel through the horizon," International Journal of Modern Physics D, vol. 13, no. 10, pp. 2351-2354, 2004.

[7] P. Kraus and F. Wilczek, "Some applications of a simple stationary line element for the Schwarzschild geometry," Modern Physics Letters A. Particles and Fields, Gravitation, Cosmology, Nuclear Physics, vol. 9, no. 40, pp. 3713-3719, 1994.

[8] P. Kraus and F. Wilczek, "Effect of self-interaction on charged black hole radiance," Nuclear Physics B, vol. 437, no. 1, pp. 231242, 1995.

[9] M. Angheben, M. Nadalini, L. Vanzo, and S. Zerbini, "Hawking radiation as tunneling for extremal and rotating black holes," Journal of High Energy Physics, vol. 2005, no. 5, p. 14, 2005.

[10] K. Srinivasan and T. Padmanabhan, "Particle production and complex path analysis," Physical Review D, vol. 60, no. 2, Article ID 024007, 20 pages, 1999.

[11] S. Shankaranarayanan, K. Srinivasan, and T. Padmanabhan, "Method of complex paths and general covariance of Hawking radiation," Modern Physics Letters A. Particles and Fields, Gravitation, Cosmology, Nuclear Physics, vol. 16, no. 9, pp. 571-578, 2001.
[12] L. Vanzo, G. Acquaviva, and R. Di Criscienzo, “Tunnelling methods and Hawking's radiation: achievements and prospects," Classical and Quantum Gravity, vol. 28, no. 18, Article ID 183001, 2011.

[13] R. Kerner and R. B. Mann, "Tunnelling, temperature, and TaubNUT black holes," Physical Review D, vol. 73, no. 10, Article ID 104010, 2006.

[14] R. Kerner and R. B. Mann, "Fermions tunnelling from black holes," Classical and Quantum Gravity, vol. 25, no. 9, Article ID 095014, 2008.

[15] R. Kerner and R. B. Mann, "Charged fermions tunnelling from kerr-newman black holes," Physics Letters B, vol. 665, no. 4, pp. 277-283, 2008.

[16] A. Yale and R. B. Mann, "Gravitinos tunneling from black holes," Physics Letters. B. Particle Physics, Nuclear Physics and Cosmology, vol. 673, no. 2, pp. 168-172, 2009.

[17] X.-Q. Li and G.-R. Chen, "Massive vector particles tunneling from Kerr and Kerr-Newman black holes," Physics Letters, Section B: Nuclear, Elementary Particle and High-Energy Physics, vol. 751, pp. 34-38, 2015.

[18] S. I. Kruglov, "Black hole emission of vector particles in (1+1)dimensions," International Journal of Modern Physics A. Particles and Fields. Gravitation. Cosmology, vol. 29, no. 22, Article ID 1450203, 2014.

[19] I. Sakalli and A. Ovgun, "Quantum tunneling of massive spin1 particles from non-stationary metrics," General Relativity and Gravitation, vol. 48, no. 1, Art. 1, 10 pages, 2016.

[20] I. Sakalli and A. Ovgun, "Tunnelling of vector particles from Lorentzian wormholes in 3+1 dimensions," European Physical Journal Plus, vol. 130, no. 6, article 110, 2015.

[21] A. Ovgun and K. Jusufi, "Massive vector particles tunneling from noncommutative charged black holes and their GUPcorrected thermodynamics," International Journal of Theoretical Physics, vol. 55, article 2919, 2016.

[22] A. Ovgun, K. Jusufi, and J. Plus, "Massive vector particles tunneling from noncommutative charged black holes and their GUP-corrected thermodynamics," The European Physical Journal Plus, vol. 131, article 177, 2016.

[23] I. Sakalli, A. Ovgun, and K. Jusufi, "GUP assisted Hawking radiation of rotating acoustic black holes," Astrophysics and Space Science. An International Journal of Astronomy, Astrophysics and Space Science, vol. 361, no. 10, 2016.

[24] K. Jusufi and A. Ovgun, "Tunneling of massive vector particles from rotating charged black strings," Astrophysics and Space Science. An International Journal of Astronomy, Astrophysics and Space Science, vol. 361, no. 7, 207 pages, 2016.

[25] K. Jusufi, "Quantum tunneling of spin-1 particles from a 5D Einstein-Yang-Mills-Gauss-Bonnet black hole beyond semiclassical approximation," EPL (Europhysics Letters), vol. 116, Article ID 60013, 2016.

[26] K. Jusufi and A. Ovgun, "Hawking radiation of scalar and vector particles from 5D myers-perry black holes," International Journal of Theoretical Physics, vol. 56, no. 6, pp. 1725-1738, 2017.

[27] B. Steven and Giddings., "Hawking radiation, the Stefan-Boltzmann law, and unitarization," Physics Letters B, vol. 754, p. 39, 2016.

[28] X.-Q. Li, "Massive vector particles tunneling from black holes influenced by the generalized uncertainty principle," Physics Letters, Section B: Nuclear, Elementary Particle and High-Energy Physics, vol. 763, pp. 80-86, 2016. 
[29] R. Li, J.-R. Ren, and D.-F. Shi, "Fermions tunneling from apparent horizon of FRW universe," Physics Letters B, vol. 670, no. 4-5, pp. 446-448, 2009.

[30] T. Zhu, J.-R. Ren, and M.-F. Li, "Corrected entropy of Friedmann-Robertson-Walker universe in tunneling method," Journal of Cosmology and Astroparticle Physics, vol. 908, no. 10, 2009.

[31] R. G. Cai, L. M. Cao, and Y. P. Hu, "Hawking radiation of an apparent horizon in a FRW universe," Classical and Quantum Gravity, vol. 26, no. 15, Article ID 155018, 2009.

[32] T. Zhu, "Hawking-like radiation as tunneling from the apparent horizon in an frw universe," International Journal of Modern Physics D, pp. 159-169, 2010.

[33] T. Jacobson, "Thermodynamics of spacetime: the Einstein equation of state," Physical Review Letters, vol. 75, p. 1260, 1995.

[34] K. Karami, A. Abdolmaleki, N. Sahraei, and S. Ghaffari, "Thermodynamics of apparent horizon in modified FRW universe with power-law corrected entropy," Journal of High Energy Physics, vol. 2011, no. 8, article no. 150, 2011.

[35] B. R. Majhi and S. Samanta, "Hawking radiation due to photon and gravitino tunneling," Annals of Physics, vol. 325, no. 11, pp. 2410-2424, 2010.

[36] H. M. Siahaan, "Higher spins tunneling from a time dependent and spherically symmetric black hole," European Physical Journal C, vol. 76, no. 3, article no. 139, 2016.

[37] M. Akbar, "Thermodynamic behavior of the Friedmann equation at the apparent horizon of the FRW universe," Physical Review D, vol. 75, Article ID 084003, 2007.

[38] M. Akbar and R.-G. Cai, "Thermodynamic behavior of field equations for $f(R)$ gravity," Physics Letters. B. Particle Physics, Nuclear Physics and Cosmology, vol. 648, no. 2-3, pp. 243-248, 2007. 

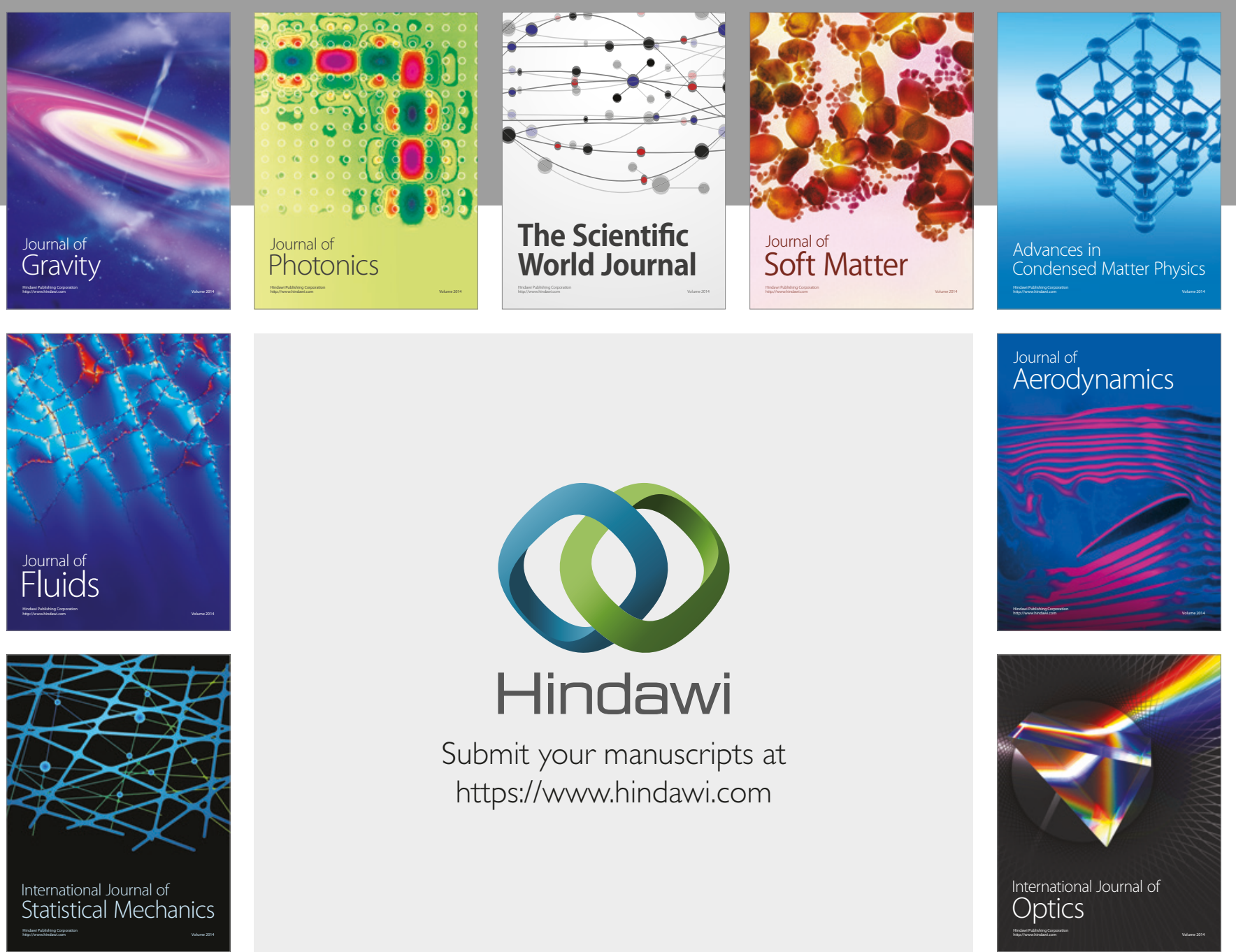

Submit your manuscripts at

https://www.hindawi.com
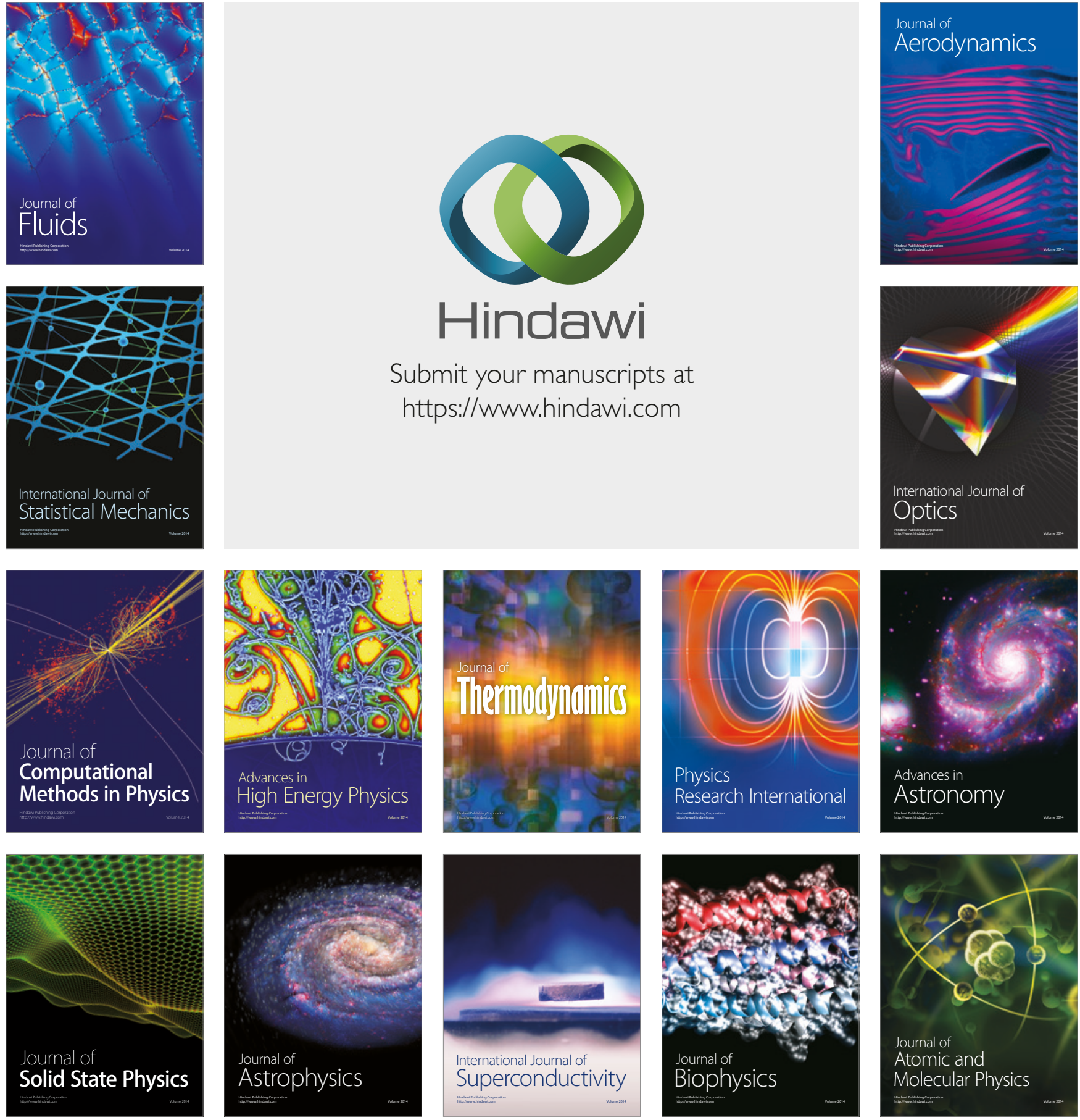